\title{
Capillary origami: superhydrophobic ribbon surfaces and liquid marbles
}

\author{
Glen McHale ${ }^{*}$, Michael I. Newton, Neil J. Shirtcliffe and Nicasio R. Geraldi
}

\section{Full Research Paper}

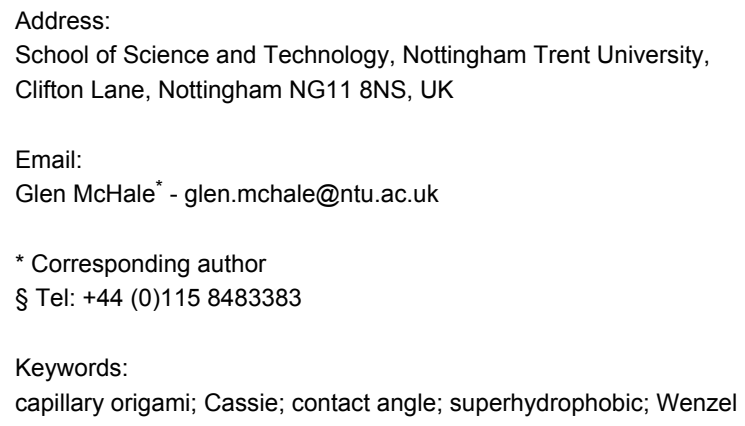

Beilstein J. Nanotechnol. 2011, 2, 145-151. doi:10.3762/bjnano.2.18

Received: 18 December 2010

Accepted: 10 February 2011

Published: 10 March 2011

This article is part of the Thematic Series "Biomimetic materials".

Guest Editors: W. Barthlott and K. Koch

(c) 2011 McHale et al; licensee Beilstein-Institut. License and terms: see end of document.

\begin{abstract}
In the wetting of a solid by a liquid it is often assumed that the substrate is rigid. However, for an elastic substrate the rigidity depends on the cube of its thickness and so reduces rapidly as the substrate becomes thinner as it approaches becoming a thin sheet. In such circumstances, it has been shown that the capillary forces caused by a contacting droplet of a liquid can shape the solid rather than the solid shaping the liquid. A substrate can be bent and folded as a (pinned) droplet evaporates or even instantaneously and spontaneously wrapped on contact with a droplet. When this effect is used to create three dimensional shapes from initially flat sheets, the effect is called capillary origami or droplet wrapping.
\end{abstract}

In this work, we consider how the conditions for the spontaneous, capillary induced, folding of a thin ribbon substrate might be altered by a rigid surface structure that, for a rigid substrate, would be expected to create Cassie-Baxter and Wenzel effects. For smooth thin substrates, droplet wrapping can occur for all liquids, including those for which the Young's law contact angle (defined by the interfacial tensions) is greater than $90^{\circ}$ and which would therefore normally be considered relatively hydrophobic. However, consideration of the balance between bending and interfacial energies suggests that the tendency for droplet wrapping can be suppressed for some liquids by providing the flexible solid surface with a rigid topographic structure. In general, it is known that when a liquid interacts with such a structure it can either fully penetrate the structure (the Wenzel case) or it can bridge between the asperities of the structure (the Cassie-Baxter case).

In this report, we show theoretically that droplet wrapping should occur with both types of solid-liquid contact. We also derive a condition for the transition between the Cassie-Baxter and Wenzel type droplet wrapping and relate it to the same transition condition known to apply to superhydrophobic surfaces. The results are given for both droplets being wrapped by thin ribbons and for solid grains encapsulating droplets to form liquid marbles. 


\section{Introduction}

In wetting, the usual implicit assumption is that a solid substrate is sufficiently thick or rigid, that it does not deform or change shape due to the interfacial forces that arise when it contacts a droplet of a liquid, however, in many natural systems this is not the case. Depositing a small droplet onto a smooth substrate and measuring the contact angle in side-profile view gives the contact angle, $\theta$, which is assumed (to within contact angle hysteresis) to approximate to the Young's law value, $\theta_{\mathrm{e}}$, given by the interfacial tensions, i.e., $\cos \theta_{\mathrm{e}}=\left(\gamma_{\mathrm{SV}}-\gamma_{\mathrm{SL}}\right) / \gamma_{\mathrm{LV}}$ where the $\gamma_{\mathrm{ij}}$ are the interfacial tensions between the solid, liquid and vapor phases. However, the bending rigidity of a solid elastic plate scales with the cube of its thickness and this assumption can become erroneous [1]. When a droplet has a radius, $R$, larger than the elastocapillary bending length [2], $L_{\mathrm{EC}}=$ $\left(\kappa_{\mathrm{b}} / \gamma_{\mathrm{LV}}\right)^{1 / 2}$ the solid can become deformed and shaped by the liquid. In practice, this effect has been given the name "capillary origami" based on experiments showing how films of polydimethylsiloxane (PDMS) shaped in two-dimensions can be folded by evaporating droplets of water to produce a designed three-dimensional shape $[3,4]$; an effect stronger than the dimpling of an elastomer surface by a deposited droplet [5]. Capillary origami is more than a curiosity and has implications for technological applications in creating three-dimensional structures from initially flat films through the capillary forces during liquid evaporation and drying [6-8]. The effect of capillary forces due to nanodroplets in activating and guiding the folding of planar graphene ribbons has recently been simulated [9].

Figure 1 illustrates capillary origami concepts and effects based on original ideas by Py et al $[3,4]$. When a PDMS (Sylgard 184) substrate of reduced thickness is contacted by a droplet of water (containing blue food dye) capillary forces bend it out of its initial planar shape (Figure 1a). When the substrate thickness is reduced to $45 \mu \mathrm{m}$ and cut into a triangular shape $(10 \mathrm{~mm}$ side lengths) and scored with a laser (Universal Laser Systems 30W $\mathrm{CO}_{2}$ laser cutter) to create fold-lines (Figure 1b), contact with a large droplet of water can create a three-dimensional shape (Figure 1d). On contact by the droplet the sheet is bent (Figure 1c) and after droplet evaporation a tetrahedron is formed (Figure 1d). Whilst this is an example of the shaping of a solid substrate by capillary forces, the final shape relies on evaporation to complete the process.

Figure 2 illustrates a number of effects as a droplet contacts a thin PDMS strip substrate ("ribbon") hanging vertically. If a droplet is deposited on a long ribbon it causes substrate deformation, but is unable to wrap or fold the substrate around itself and, as evaporation proceeds, the deformation decreases (Figure 2a). However, when the length of ribbon below the
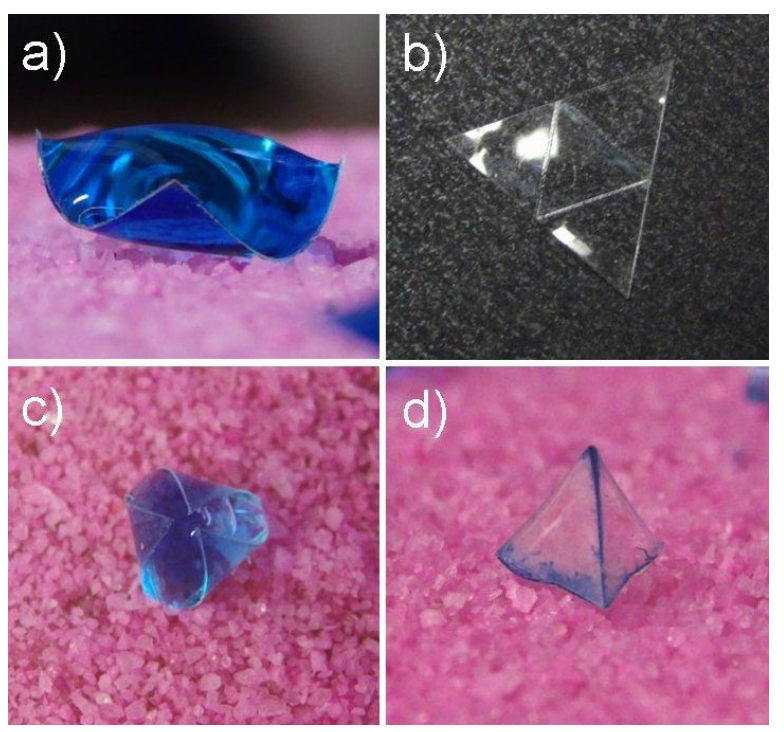

Figure 1: Effect of droplets of blue-dyed water on a thin polydimethylsiloxane (PDMS) membrane: a) droplet causing bending of the substrate, $b$ ) initial shaped substrate with the three score lines for folding, c) droplet induced folding, and d) three-dimensional shape left after completion of evaporation.

droplet contact point is sufficiently short, the contacting droplet can quickly fold the ribbon up against gravity and wrap itself. Figure 1 and Figure 2 are illustrative of the ability of capillary forces to deform, fold and bend substrates. The concepts of capillary origami and droplet wrapping also have implications for our understanding of the definition of hydrophobicity and its relationship to adhesion. Gao and McCarthy demonstrated that spontaneous and complete droplet wrapping occurs, without the need for evaporation, with a thin film of Teflon ${ }^{\circledR}$ even though this material would normally have a contact angle to water greater than $90^{\circ}$ and so be regarded as hydrophobic [10]; an effect one of the current authors explained on the basis of the changes in the balance between interfacial and bending energies [11].

In a previous report, McHale argued from surface free energy considerations that, when the bending energy is small, all solids should demonstrate droplet wrapping and so can, in an absolute sense, be considered hydrophilic [11]. That work also discussed why for a partially wetting droplet to be observed there is necessarily an assumption of some rigidity of the substrate, so that the usual definition of relative hydrophobicity (and relative hydrophilicity) through contact angle measurement includes a structural non-surface chemistry based assumption about the solid. It was also suggested that a set of loose spherical grains could be considered to be the extreme case of a solid with no bending energy, thus relating the concept of droplet wrapping to 


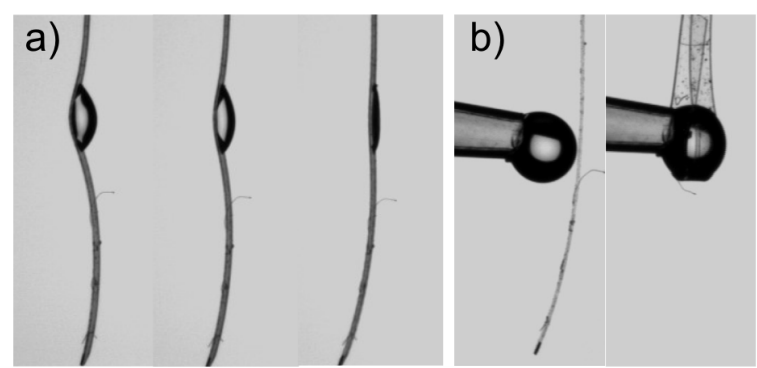

Figure 2: Effect of droplets of water on a thin polydimethylsiloxane (PDMS) membrane ribbon substrate hanging vertically: a) droplet causing a bending of the substrate which disappears as evaporation proceeds (three frames), b) spontaneous wrapping as a droplet touches a membrane ribbon (initial and final states). that of the formation of liquid marbles [12,13]. It was further argued that when the flexible solid surface possessed rigid surface roughness or the solid grains had a rigid surface roughness, droplet wrapping might, under defined conditions for the surface chemistry defined contact angle, be suppressed. Since wrapping a spherical droplet requires both bending and stretching of the solid, in this report, we consider the simpler, but experimentally realizable, cases of wrapping of a droplet of water by a thin ribbon and the assembly of solid grains to form a liquid marble. For both cases, we extend the previous theoretical consideration to ribbon-type substrates and disconnected solid grains with a rigid surface structure. We review the case for surface roughness that has low aspect ratio so that the liquid can penetrate into the structure - the Wenzel case $[14,15]$. We then consider whether droplet wrapping can occur without penetration into the surface structure - the Cassie-Baxter case $[16,17]$. We show that droplet wrapping should occur with both types of configuration and we derive a condition for the transition between these two cases; this condition is the same as for the Wenzel to Cassie-Baxter transition on a superhydrophobic surface $[18,19]$.

\section{Results and Discussion}

\section{Droplet wrapping theory}

To assess whether it is energetically favourable for a liquid to become wrapped in a solid we consider the change in interfacial energy as the solid-vapor interface is replaced by a solid-liquid interface together with the increase in bending energy as the solid deforms from a planar ribbon, similar to those shown in Figure 2, of width $w<<R$, where $R$ is the droplet radius. The use of a ribbon substrate allows the problem to be simplified to a quasi-two dimensional situation. Assuming there is no spontaneous curvature of the solid film, the initial energy is given by the sum of the energy associated with the liquid in contact with the vapor and the surfaces of the solid in contact with the vapor (Figure 3a),

$$
F^{\mathrm{i}}=A_{\mathrm{LV}}^{\mathrm{i}} \gamma_{\mathrm{LV}}+r_{\mathrm{W}} A_{\mathrm{SV}}^{\mathrm{p}} \gamma_{\mathrm{SV}}+A_{\mathrm{SV}}^{\text {lower }} \gamma_{\mathrm{SV}}
$$

where $A_{\mathrm{LV}}^{\mathrm{i}}$ is the initial liquid-vapor interfacial area, $A^{\mathrm{p}} \mathrm{SV}$ is the initial planar projection of the area of the upper surface of the solid film, $r_{\mathrm{W}}$ is the Wenzel roughness of the surface, and the $\gamma_{\mathrm{ij}}$ are the interfacial tensions; the lower surface of the film is assumed to have an area $A^{\text {lower }} \mathrm{SV}$. The initial liquid-vapor area is $A_{\mathrm{LV}}^{\mathrm{i}}=4 \pi R^{2}$, where $R$ is the droplet radius, and after wrapping it is assumed that the shape is spherical with the same radius $R$. This means that a planar projected area $2 \pi R w$ of the ribbon's area is involved in the wrapping. For simplicity in the following, we limit the initial ribbon length to $2 \pi R$, so that $A^{\mathrm{p}_{\mathrm{SV}}}$ $=2 \pi R w$ is assumed.

\section{a) Initial state}

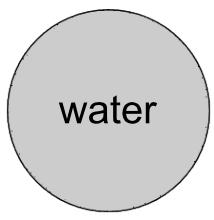

vapor

solid
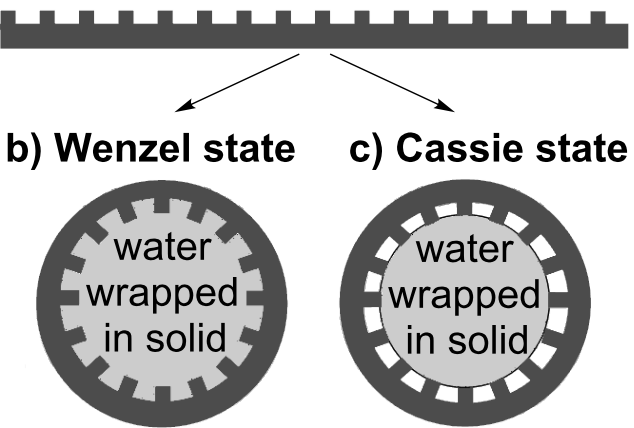

Figure 3: Initial and final states involved in a droplet wrapping event for a flexible ribbon membrane with rigid roughness. In the Wenzel case the liquid penetrates between features and in the Cassie case it bridges between them.

The energy per unit area, $f_{\mathrm{b}}$, associated with bending and stretching a thin membrane substrate is related to the principal radii of curvatures of the substrate,

$$
f_{\mathrm{b}}=\frac{\kappa_{\mathrm{b}}\left(c_{1}+c_{2}\right)^{2}}{2}+\kappa_{\mathrm{G}} c_{1} c_{2}
$$

where $\kappa_{\mathrm{b}}$ is the elastic bending rigidity and $\kappa_{\mathrm{G}}$ is the Gaussian bending modulus [20]. For a film of thickness $h$, the bending rigidity is given by $\kappa_{\mathrm{b}}=E h^{3} / 12\left(1-v^{2}\right)$, where $E$ is Young's modulus and $v$ is Poisson's ratio; the Gaussian bending modulus relates to any stretching or compression of the film. The coefficients $c_{1}$ and $c_{2}$ are the principal radii of curvature, which for a 
spherical droplet are $c_{1}=c_{2}=1 / R$. For a ribbon bending only along its length $c_{1}=1 / R$ and $c_{2}=0$ so that for a radius of $R$ the bending energy per unit area is,

$$
f_{\mathrm{b}}=\frac{\kappa_{\mathrm{b}} c_{1}^{2}}{2}=\frac{\kappa_{\mathrm{b}}}{2 R^{2}}
$$

When the liquid comes into contact with the ribbon, assuming the ribbon can bend, and that the roughness remains unchanged, we can imagine two types of wrapping scenarios. In the Wenzel case, the liquid may penetrate between the surface features and retain contact with the ribbon at all points along its surface (Figure 3b). In the alternative Cassie-Baxter case, the surface structure combined with the surface chemistry may be such that the liquid bridges between the tops of the surface features leaving vapor between them (Figure $3 \mathrm{c}$ ).

\subsection{Wenzel case}

In the Wenzel case, the liquid penetrates between surface features (Figure $3 b$ ) and the difference in energy between the final and the initial state related to the attachment of the droplet to the ribbon is given by,

$$
\frac{\Delta F_{\mathrm{W}}}{2 \pi R w}=r_{\mathrm{W}}\left(\gamma_{\mathrm{SL}}-\gamma_{\mathrm{SV}}\right)-\gamma_{\mathrm{LV}}+\frac{\kappa_{\mathrm{b}} c_{1}^{2}}{2}
$$

which can be rewritten using the definition of the Young's law equilibrium contact angle on a rigid surface of $\cos \theta_{\mathrm{e}}=\left(\gamma_{\mathrm{SV}}-\right.$ $\left.\gamma_{\mathrm{SL}}\right) / \gamma_{\mathrm{LV}}$, as,

$$
\frac{\Delta F_{\mathrm{W}}}{2 \pi R w}=-\gamma_{\mathrm{LV}}\left(r_{\mathrm{W}} \cos \theta_{\mathrm{e}}+1\right)+\frac{\kappa_{\mathrm{b}} c_{1}^{2}}{2}
$$

For liquids which on a rigid smooth solid substrate are considered to be partially wetting the $\operatorname{cosine}$ satisfies $-1<\cos \theta_{\mathrm{e}}<1$ and $\theta_{\mathrm{e}}$ gives a finite Young's law contact angle. However, for those liquids which completely wet and form films, the combination $\left(\gamma_{S V}-\gamma_{S L}\right) / \gamma_{L V}$ has a value greater than 1 . The combination of the roughness, $r_{\mathrm{W}}$, multiplying $\cos \theta_{\mathrm{e}}$ immediately introduces the Wenzel contact angle,

$$
\frac{\Delta F_{\mathrm{W}}}{2 \pi R w}=-\gamma_{\mathrm{LV}}\left(\cos \theta_{\mathrm{W}}+1\right)+\frac{\kappa_{\mathrm{b}} c_{1}^{2}}{2}
$$

One assumption in Equation 6 is that the final radius of the wrapped portion of the droplet is approximately the same as the initial droplet radius.

\subsection{Cassie-Baxter case}

In the Cassie-Baxter case, complete penetration of liquid between surface features does not occur (Figure 3c). The liquid only contacts a fraction $\varphi_{\mathrm{S}}$ of the surface thus leaving a fraction $\left(r_{\mathrm{W}}-\varphi_{\mathrm{S}}\right)$ of the solid surface in contact with the vapor. In addition, the liquid bridges between surface features, thus providing a set of menisci, here approximated by a fraction $\left(1-\varphi_{\mathrm{s}}\right)$ of the surface with a liquid-vapor interface. The difference in energy between the final and the initial state related to the attachment of the droplet to the ribbon is then given by,

$$
\begin{aligned}
\frac{\Delta F_{\mathrm{CB}}}{2 \pi R w} & =\varphi_{\mathrm{s}} \gamma_{\mathrm{SL}}+\left(1-\varphi_{\mathrm{S}}\right) \gamma_{\mathrm{LV}}+ \\
& \left(r_{\mathrm{W}}-\varphi_{\mathrm{S}}\right) \gamma_{\mathrm{SV}}-r_{\mathrm{W}} \gamma_{\mathrm{SV}}-\gamma_{\mathrm{LV}}+\frac{\kappa_{\mathrm{b}} c_{1}^{2}}{2}
\end{aligned}
$$

Cancelling terms involving the roughness factor $r_{\mathrm{W}}$ and using the definition of the equilibrium contact angle on a rigid substrate of $\cos \theta_{\mathrm{e}}=\left(\gamma_{\mathrm{SV}}-\gamma_{\mathrm{SL}}\right) / \gamma_{\mathrm{LV}}$ gives,

$$
\frac{\Delta F_{\mathrm{CB}}}{2 \pi R w}=-\gamma_{\mathrm{LV}}\left(\varphi_{\mathrm{s}} \cos \theta_{\mathrm{e}}-\left(1-\varphi_{\mathrm{s}}\right)+1\right)+\frac{\kappa_{\mathrm{b}} c_{1}^{2}}{2}
$$

Defining the Cassie-Baxter combination $\cos \theta_{\mathrm{CB}}=\varphi_{\mathrm{s}} \cos \theta_{\mathrm{e}}-$ $\left(1-\varphi_{\mathrm{s}}\right)$, which is familiar from the modelling of droplets on superhydrophobic surfaces, gives,

$$
\frac{\Delta F_{\mathrm{CB}}}{2 \pi R w}=-\gamma_{\mathrm{LV}}\left(\cos \theta_{\mathrm{CB}}+1\right)+\frac{\kappa_{\mathrm{b}} c_{1}^{2}}{2}
$$

The similarity of Equation 6 and Equation 9 can be revealed by writing,

$$
\frac{\Delta F_{\mathrm{T}}}{2 \pi R w}=-\gamma_{\mathrm{LV}}\left(\cos \theta_{\mathrm{T}}+1\right)+\frac{\kappa_{\mathrm{b}} c_{1}^{2}}{2}
$$

where the subscript $\mathrm{T}$ defines the topographic assumption of the liquid either in a Wenzel ("penetrating") or Cassie-Baxter ("skating") state. In the form presented by Equation 10, the principal radius of curvature $c_{1}$ is given by $1 / R$ and so the energy change per unit area of the ribbon substrate depends on the droplet size.

\subsection{Wrapping and transitions with roughness}

The wrapping state will be stable provided the energy change given by Equation 10 is negative, i.e., 


$$
\cos \theta_{\mathrm{T}}>-1+\frac{\kappa_{\mathrm{b}} c_{1}^{2}}{2 \gamma_{\mathrm{LV}}}
$$

Defining the dimensionless curvature elastocapillary number $n_{\mathrm{EC}}=\kappa_{\mathrm{b}} c_{1}^{2} / 2 \gamma_{\mathrm{LV}}$, Equation 11 can be written as,

$$
\cos \theta_{\mathrm{T}}>-1+n_{\mathrm{EC}}
$$

A ribbon substrate that is unable to bend in response to contact with the liquid will have an elastocapillary number that tends to infinity and so wrapping will not occur. When the elastocapillary number has a finite value, wrapping will occur, but will depend on the volume and shape of the liquid. For a droplet with a spherical shape of radius $R$, the elastocapillary number is $n_{\mathrm{EC}}=\kappa_{\mathrm{b}} / 2 \gamma_{\mathrm{LV}} R^{2}=1 / 2\left(L_{\mathrm{EC}} / R\right)^{2}$, where $L_{\mathrm{EC}}=$ $\left(\kappa_{\mathrm{b}} / \gamma_{\mathrm{LV}}\right)^{1 / 2}$ is the characteristic elastocapillary length. Equation 12 then becomes,

$$
\cos \theta_{\mathrm{T}}>-1+2\left(\frac{L_{\mathrm{EC}}}{R}\right)^{1 / 2}
$$

This condition for droplet wrapping depends upon the state of contact of the wrapped liquid with the solid surface, i.e., penetrating or skating. For the Cassie-Baxter state with its air-pockets to be thermodynamically stable compared to the Wenzel state, requires $\Delta F_{\mathrm{CB}}<\Delta F_{\mathrm{W}}$ in addition to $\Delta F_{\mathrm{CB}}<0$. Since the curvature energy contributes the same to both, Equation 10 implies $\cos \theta_{\mathrm{W}}<\cos \theta_{\mathrm{CB}}$, which gives a condition on the relationship between the Young's law contact angle $\theta_{\mathrm{e}}$, and the roughness $r_{\mathrm{W}}$ and solid surface fraction $\varphi_{\mathrm{s}}$,

$$
\cos \theta_{\mathrm{e}}<-\left(\frac{1-\varphi_{\mathrm{s}}}{r_{\mathrm{W}}-\varphi_{\mathrm{s}}}\right)=\cos \theta_{\mathrm{c}}
$$

where $\theta_{\mathrm{c}}$ is a critical contact angle for thermodynamic stability of the Cassie-Baxter state; when the Young's law contact angle exceeds the critical contact angle the Cassie-Baxter state is favoured over the Wenzel state. Equation 14 is exactly the same as the condition derived by Bico et al., for the thermodynamic stability of the Cassie-Baxter state on a superhydrophobic surface $[18,19]$. As noted by these authors, when $90^{\circ}<\theta_{\mathrm{e}}<\theta_{\mathrm{c}}$ the Cassie-Baxter state may exist due to, e.g., pinning on sharp edges of features, but it is a metastable state.

Here we have also only considered a simple model that assumes either a Wenzel state or a Cassie-Baxter state. However, surfaces with curvature can effectively have a combination of both Wenzel and Cassie-Baxter properties with the solid surface fraction becoming a function of the Young's law contact angle [21]. Re-entrant surfaces have been shown to be particularly effective in producing suspended droplets of liquids with low surface tensions [22]. Following the superhydrophobic literature, we can also anticipate that if the surface chemistry tends towards hydrophilic (i.e., $\theta_{\mathrm{e}}<90^{\circ}$ ) there might be a hemi-wicking effect with the liquid invading the surface texture, but wetting the asperities of the topographic features. A simple two-dimensional model consideration of the energy changes as a liquid invades a structure on a thin substrate suggests that the critical Young's law contact angle for hemiwicking will be shifted to values lower than $\theta_{\mathrm{c}}$ due to the contribution of bending energy.

\subsection{Drop size and contact angle effects}

The inclusion of the energy associated with the curvature of a substrate introduces a characteristic elastocapillary length and results in drop size effects. For a ribbon film substrate, Equation 10 implies wrapping requires the droplet radius $R$ to be greater than a critical radius, $R_{\mathrm{c}}$, given by,

$$
R_{\mathrm{c}}=\left(\frac{\kappa_{\mathrm{b}}}{2 \gamma_{\mathrm{LV}}\left(\cos \theta_{\mathrm{T}}+1\right)}\right)^{1 / 2}=\left(\frac{1}{2\left(\cos \theta_{\mathrm{T}}+1\right)}\right)^{1 / 2} L_{\mathrm{EC}}
$$

which can be compared to the condition $R>L_{\mathrm{EC}} / \sqrt{ } 2$ given by Py et al [3]. Thus, there is a critical radius which depends on the Young's law contact angle, $\theta_{\mathrm{e}}$, and the topographic structure via the surface roughness, $r_{\mathrm{W}}$, or solid surface fraction, $\varphi_{\mathrm{s}}$.

In the Cassie-Baxter case, $\cos \theta_{\mathrm{T}}=\cos \theta_{\mathrm{CB}}$, and $\theta_{\mathrm{CB}}$ can approach $180^{\circ}$ from below and, as it does so, the critical radius for wrapping tends to infinity; a strongly superhydrophobic ribbon will not result in droplet wrapping because the energy gain cannot overcome the bending energy. In the Wenzel case, $\cos \theta_{\mathrm{T}}$ $=\cos \theta_{\mathrm{W}}$, and this is positive when $\theta_{\mathrm{e}}<90^{\circ}$, but negative when $\theta_{\mathrm{e}}>90^{\circ}$. In the former case, the critical radius becomes smaller as the Young's law contact angle tends to zero or as the roughness increases; a film can be wrapped in a tighter curve and, hence, a smaller droplet radius is needed. It should also be noted that $\cos \theta_{\mathrm{e}}$ is defined by a combination of the interfacial tensions and this combination can be greater than unity; this corresponds to a film of liquid on a smooth and rigidly flat surface. In the considerations above, no account has been taken of the finite mass of the substrate on the critical volume of liquid required for wrapping; a problem recently considered experimentally and theoretically for square and triangular sheets of PDMS by Chen et al [23]. 


\section{Liquid marbles and topographically struc- tured grains}

When a solid in the form of a thin ribbon wraps around a droplet it only needs to bend, whereas when the solid is a sheet it needs to either stretch and deform or to crumple and fold. Such a situation could be considered, but additional energies relating to these effects would need to be included unless the contribution from these is at no energy cost. One possible situation that conceptually is similar to a substrate able to deform and conform to a liquid surface, but without any bending or stretching energy cost, is the adhesion of a collection of solid grains to a liquid surface to encapsulate it and form a liquid marble (Figure 4a and Figure 4b) $[12,13,24]$. In an abstract sense, a collection of grains assembled in a close-packed form onto a liquid-vapor interface is the extreme limit of a flexible solid possessing no curvature (or stretching) energy and, hence, a vanishing elastocapillary length. In the study of liquid marbles, the simplest assumption is that each grain is spherical in shape and has no particular surface topography. As a consequence all grains, irrespective of their surface chemistry, will adhere to the water-air interface; a similar conclusion to that regarding the absolute hydrophilicity of solids when their curvature energy is zero. The effect of surface chemistry, characterised through the Young's law contact angle, is to determine the strength of the adhesion to the air-water interface with maximum strength corresponding to $\theta_{\mathrm{e}}=90^{\circ}$; if $\theta_{\mathrm{e}}>90^{\circ}$ more than half the grain projects out of the interface into the air. In practice, the surfaces of the grains do not need to be smooth and can have a topographic structure. For example, pollen grains come in a variety of shapes, commonly spherical, ovoid or disclike with lengths in the order of $10-100 \mu \mathrm{m}$ and their surfaces (exine) under scanning electron microscopy vary from relatively smooth to mesh-like and ones adorned with sharp spikes (see, e.g., [25]).

By considering the changes in interfacial areas as a spherical grain of radius $R_{\mathrm{g}}$ with a roughness $r_{\mathrm{W}}$ attaches to a droplet of radius $R$ (Figure $4 \mathrm{c}$ ), we deduce the change in surface free energy $\Delta F^{\mathrm{M}}$,

$$
\Delta F_{\mathrm{T}}^{\mathrm{M}}=-A_{\text {cap }} \gamma_{\mathrm{LV}}\left(1+\cos \theta_{\mathrm{T}}\right)
$$

where $A_{\text {cap }}=\pi R_{\mathrm{g}}{ }^{2}\left(1+\cos \theta_{\mathrm{T}}\right)$ is the spherical cap area of the solid grain of radius $R_{\mathrm{g}}$ intersecting the droplet and $\theta_{\mathrm{T}}$ is either the Wenzel contact angle or the Cassie-Baxter contact angle, depending on whether the liquid penetrates between the topographic features on the surface of the grain or whether it bridges between the asperities (and is therefore only in contact with a fraction of the solid area, $\varphi_{\mathrm{s}}$ ). In a similar manner to droplet wrapping, Equation 14 defines a minimum Young's law contact

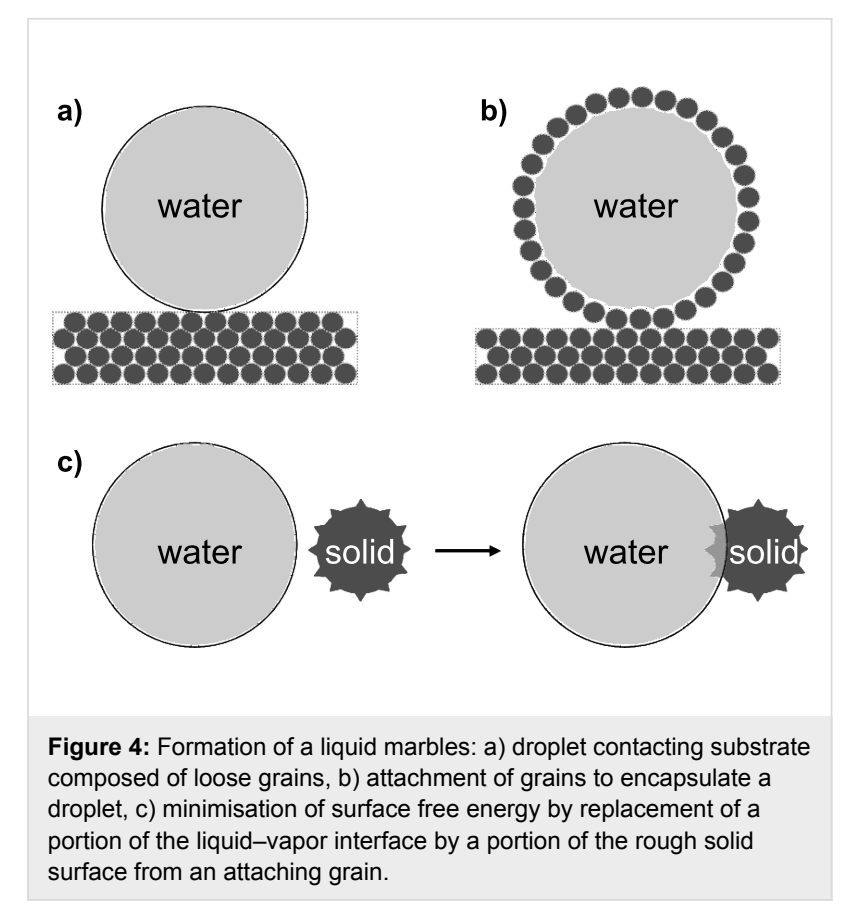

angle for the Cassie-Baxter state to be thermodynamically stable over the Wenzel state. The idea of a solid film that tends to a non-adhesive surface for liquids can be extended to nonstick granular or powder systems. All smooth spherical grains adhere to the liquid interface because $\left(1+\cos \theta_{\mathrm{e}}\right)$ can never be negative. However, when the surface of a grain is structured it can become superhydrophobic and it will then only weakly attach to the surface of the liquid.

\section{Conclusion}

In this work, we have focused on a rigid surface structure on a thin flexible substrate, but the inverse situation of a flexible surface structure on a rigid substrate has recently also been modelled [26,27]. A result of that work is an understanding that elastocapillary effects can provide additional stability for Cassie-type suspended liquid states involved in, e.g., plastron respiration [27-29]. It therefore seems likely that to fully understand superhydrophobic surfaces, the flexible nature of elements of surfaces needs to be understood. Using a model of a thin ribbon (strip) substrate we have shown that relaxing the assumption of a rigid substrate allows a contacting droplet to shape and bend the substrate provided the droplet radius is larger than a critical value. When the flexible substrate has a surface with a rigid topographic structure, the critical droplet radius at which droplets wrap depends on both the elastocapillary length and a function of either the Wenzel or the Cassie-Baxter contact angle dependent on the state of the contact. We have argued that liquid marbles can be thought of as such a system, but with a vanishing elastocapillary length. Manipulating the surface structure therefore provides a method, 
complementary to control of substrate thickness, to tune the balance of adhesive forces between liquids and solids both within capillary origami and granular systems.

\section{Acknowledgements}

One of the authors (NG) is grateful to Nottingham Trent University for providing a $\mathrm{PhD}$ scholarship.

\section{References}

1. Olives, J. J. Phys.: Condens. Matter 1993, 5, 2081-2094. doi:10.1088/0953-8984/5/14/007

2. Bico, J.; Roman, B.; Moulin, L.; Boudaoud, A. Nature 2004, 432, 690. doi:10.1038/432690a

3. Py, C.; Reverdy, P.; Doppler, L.; Bico, J.; Roman, B.; Baroud, C. N. Phys. Rev. Lett. 2007, 98, No. 156103. doi:10.1103/PhysRevLett.98.156103

4. Py, C.; Reverdy, P.; Doppler, L.; Bico, J.; Roman, B.; Baroud, C. N Eur. Phys. J. Special Topics 2009, 166, 67-71. doi:10.1140/epjst/e2009-00880-4

5. Pericet-Camara, R.; Auernhammer, G. K.; Koynov, K.; Lorenzoni, S.; Raiteri, R.; Bonaccurso, E. Soft Matter 2009, 5, 3611-3617. doi:10.1039/b907212h

6. De Volder, M.; Tawfick, S. H.; Park, S. J.; Copic, D.; Zhao, Z.; Lu, W.; Hart, A. J. Adv. Mater. 2010, 22, 4384-4389. doi:10.1002/adma.201001893

7. Ahn, B. Y.; Shoji, D.; Hansen, C. J.; Hong, E.; Dunand, D. C.; Lewis, J. A. Adv. Mater. 2010, 22, 2251-2254. doi:10.1002/adma.200904232

8. van Honschoten, J. W.; Berenschot, J. W.; Ondarçuhu, T.; Sanders, R. G. P.; Sundaram, J.; Elwenspoek, M.; Tas, N. R. Appl. Phys. Lett. 2010, 97, No. 014103. doi:10.1063/1.3462302

9. Patra, N.; Wang, B.; Král, P. Nano Lett. 2009, 9, 3766-3771. doi:10.1021/nl9019616

10. Gao, L.; McCarthy, T. J. Langmuir 2008, 24, 9183-9188. doi:10.1021/la8014578

11. McHale, G. Langmuir 2009, 25, 7185-7187. doi:10.1021/la900597a

12. Aussillous, P.; Quéré, D. Nature (London) 2001, 411, 924-927. doi:10.1038/35082026

13. McHale, G.; Herbertson, D. L.; Elliott, S. J.; Shirtcliffe, N. J.; Newton, M. I. Langmuir 2007, 23, 918-924. doi:10.1021/la061920j

14. Wenzel, R. N. Ind. Eng. Chem. 1936, 28, 988-994. doi:10.1021/ie50320a024

15. Wenzel, R. N. J. Phys. Colloid Chem. 1949, 53, 1466-1467. doi:10.1021/j150474a015

16. Cassie, A. B. D.; Baxter, S. Trans. Faraday Soc. 1944, 40, 546-551. doi:10.1039/tf9444000546

17. Cassie, A. B. D. Discuss. Faraday Soc. 1948, 3, 11-16. doi:10.1039/df9480300011

18. Bico, J.; Thiele, U.; Quéré, D. Colloids Surf., A 2002, 206, 41-46. doi:10.1016/S0927-7757(02)00061-4

19. Quéré, D. Annu. Rev. Mater. Res. 2008, 38, 71-99. doi:10.1146/annurev.matsci.38.060407.132434

20. Boal, D. H. Mechanics of the Cell; Cambridge University Press: New York, 2002.

21. Shirtcliffe, N. J.; McHale, G.; Atherton, S.; Newton, M. I. Adv. Colloid Interface Sci. 2010, 161, 124-138. doi:10.1016/j.cis.2009.11.001
22. Tuteja, A.; Choi, W.; Ma, M.; Mabry, J. M.; Mazzella, S. A.; Rutledge, G. C.; McKinley, G. H.; Cohen, R. E. Science 2007, 318, 1618-1622. doi:10.1126/science.1148326

23. Chen, L.; Wang, X.; Wen, W.; Li, Z. Appl. Phys. Lett. 2010, 97, No. 124103. doi:10.1063/1.3492834

24. Shirtcliffe, N. J.; McHale, G.; Newton, M. I.; Pyatt, F. B.; Doerr, S. H. Appl. Phys. Lett. 2006, 89, No. 094101. doi:10.1063/1.2339072

25. Shaheen, N.; Khan, M. A.; Hayat, M. Q.; Yasmin, G. J. Med. Plants Res. 2009, 3.

26. Bernardino, N. R.; Blickle, V.; Dietrich, S. Langmuir 2010, 26, 7233-7241. doi:10.1021/la904345r

27. Blow, M. L.; Yeomans, J. M. Langmuir 2010, 26, 16071-16083. doi:10.1021/la101847b

28. McHale, G.; Newton, M. I.; Shirtcliffe, N. J. Soft Matter 2010, 6, 714-719. doi:10.1039/b917861a

29. Shirtcliffe, N. J.; McHale, G.; Newton, M. I.; Perry, C. C.; Pyatt, F. B. Appl. Phys. Lett. 2006, 89, No. 104600. doi:10.1063/1.2347266

\section{License and Terms}

This is an Open Access article under the terms of the Creative Commons Attribution License

(http://creativecommons.org/licenses/by/2.0), which permits unrestricted use, distribution, and reproduction in any medium, provided the original work is properly cited.

The license is subject to the Beilstein Journal of

Nanotechnology terms and conditions:

(http://www.beilstein-journals.org/bjnano)

The definitive version of this article is the electronic one which can be found at: doi:10.3762/bjnano.2.18 